\title{
Direct quantification of $3^{\prime}$ terminal 2'-O-methylation of small RNAs by RT-qPCR
}

\author{
NAN WANG,${ }^{1,4}$ SHUANG QU, ${ }^{1,4}$ WU SUN, ${ }^{1,2,4}$ ZIYI ZENG, ${ }^{3}$ HONGWEI LIANG, ${ }^{1}$ CHEN-YU ZHANG, ${ }^{1}$ \\ XI CHEN, ${ }^{1}$ and KE ZEN ${ }^{1}$ \\ ${ }^{1}$ State Key Laboratory of Pharmaceutical Biotechnology, Nanjing Advanced Institute for Life Sciences (NAILS), Nanjing University, Nanjing, \\ Jiangsu, 210093, China \\ ${ }^{2}$ Tianjin Medical University Cancer Institute and Hospital, National Clinical Research Center for Cancer, Key Laboratory of Cancer Prevention \\ and Therapy, Tianjin's Clinical Research Center for Cancer, Tianjin 300060, China \\ ${ }^{3}$ School of Life Science and Medicine, Dalian University of Technology Panjin Campus, Panjin, Liaoning, 124000, China
}

\begin{abstract}
Modification of nucleotides significantly increases the diversity of functional nucleic acids. As one of the most common modifications of RNAs, methylation of the 2'-hydroxyl-group of ribonucleotides (2'-O-methylation) has been found in various RNAs in eukaryotes. However, due to the lack of an efficient method for quantifying small RNA $3^{\prime}$ terminal $2^{\prime}-$ O-methylation, it is difficult to monitor the dynamic change of $3^{\prime}$ terminal $2^{\prime}$-O-methylation during various biological processes. Capitalizing on the finding that $3^{\prime}$ terminal RNA 2'-O-methylation can inhibit the activity of poly(A) polymerase, an enzyme that can add the poly(A)-tail to RNA, we develop a method by which the 2'-O-methylation level of small RNAs, such as microRNAs (miRNAs) and Piwi-interacting RNAs (piRNAs), can be directly quantified based on the poly(A)-tailed RT-qPCR technique. With this method, we successfully determine the 2'-O-methylation level of miRNAs in Arabidopsis thaliana and mouse lung tissue, piRNA in human seminal plasma, and monitor the alteration of miRNA 2'-O-methylation in Drosophila Schneider 2 cells after knockdown of Drosophila methyltransferase protein Hua enhancer 1 (DmHen-1).
\end{abstract}

Keywords: methylation; RT-qPCR; miRNA

\section{INTRODUCTION}

Nucleic acid modification is ubiquitous across many species and yields diversification of DNA and RNA (Limbach et al. 1994). Since the first pseudouridine was discovered 50 yr ago (Cohn 1960), more than 100 different RNA modifications have been identified in different organisms (Motorin and Helm 2011; Machnicka et al. 2013). Modified RNA molecules include tRNA, rRNA, mRNA, and a variety of small RNAs such as plant microRNAs (miRNAs). Among these various modifications, 2'-O-methylation (2'Ome), found in various RNAs in eukaryotes as well as bacteria and archaea, is one of the most common modifications of RNA. Recent studies indicate that different small RNAs in insects and mammals, including small interfering RNAs (siRNAs) and piRNAs, have $3^{\prime}$ terminal 2'Ome (Kirino and Mourelatos 2007a; Shen et al. 2012).

It has been suggested that methyltransferase protein Hua enhancer 1 (HEN1) mediates the $3^{\prime}$ terminal $2^{\prime}$ Ome

\footnotetext{
${ }^{4}$ These authors contributed equally to this work.

Corresponding authors: kzen@nju.edu.cn, xichen@nju.edu.cn, cyzhang@nju.edu.cn

Article is online at http://www.rnajournal.org/cgi/doi/10.1261/rna. 065144.117.
}

of miRNAs, siRNAs, and piRNAs (Horwich et al. 2007; Kirino and Mourelatos 2007b; Ohara et al. 2007; Saito et al. 2007). In plants, nearly all small RNAs are 2'-O-methylated, regardless of the $A G O$ proteins to which they bind (Yang et al. 2006). Arabidopsis HEN1 mediates the methylation of double-stranded small RNAs before their loading into AGO proteins (Yu et al. 2005). In Drosophila, the termini of siRNAs and piRNAs is methylated by DmHen1, the Drosophila homolog of HEN1 (Ghildiyal and Zamore 2009). Unlike plant HEN1, DmHen1 acts on a single strand in RISC but not on an RNA duplex (Horwich et al. 2007). Also in Drosophila, the small RNAs bearing the $2^{\prime}$-O-methylation at their $3^{\prime}$ terminal end were generally found to be bound to AGO2 instead of AGO1 (Ameres et al. 2010). Although the exact role of $3^{\prime}$ terminal $2^{\prime}$ Ome for small RNAs remains unclear, it has been shown that $3^{\prime}$ terminal $2^{\prime}$ Ome can protect small RNAs from the $3^{\prime}$ end uridylation and $3^{\prime}$ to $5^{\prime}$ exonucleolytic degradation (Li et al. 2005; Ji

C 2018 Wang et al. This article is distributed exclusively by the RNA Society for the first 12 months after the full-issue publication date (see http://rnajournal.cshlp.org/site/misc/terms.xhtml). After 12 months, it is available under a Creative Commons License (Attribution-NonCommercial 4.0 International), as described at http://creativecommons.org/ licenses/by-nc/4.0/. 
and Chen 2012). A previous study shows that poxvirusesencoded poly $(A)$ polymerase mediates $3^{\prime}$ polyadenylation of host miRNAs, resulting in the degradation of miRNAs (Backes et al. 2012), and $3^{\prime}$ end 2'Ome may protect small RNAs from degradation during viral infection.

Although 2'Ome methylation is the most common modifications of RNAs, it is difficult to quantify the level of 2 'Ome methylation in small RNAs including miRNAs and piRNAs (Aschenbrenner and Marx 2016). Presently, the identification of small RNA 2'Ome mainly relies on sodium periodate oxidation and northern blot or mass spectrometry (Yu et al. 2005; Ohara et al. 2007). These methods do identify the presence of $2^{\prime}$ Ome in small RNAs but yield little quantitative information. Moreover, these technologies are labor intensive and time-consuming. Although a direct and site-specific quantification of RNA 2'Ome has been recently developed for long RNA fragments such as rRNA (Aschenbrenner and Marx 2016), there is no method available for directly quantifying the $3^{\prime}$ terminal $2^{\prime}$ Ome of small RNAs. With the observation of the ubiquity of $3^{\prime}$ terminal $2^{\prime}$ Ome modifications across a wide variety of RNAs, there is an urgent need for a direct method to quantify the $3^{\prime}$ terminal 2'Ome level of small RNAs.

In the present study, we capitalize on the observation that 2'Ome of the terminal nucleotide can inhibit the activity of poly(A) polymerase and develop a poly(A)-tail RTQPCR method for directly quantifying the $3^{\prime}$ terminal $2^{\prime}$ Ome level of small RNAs. Using this novel method, we have accurately quantified the level of $3^{\prime}$ terminal $2^{\prime}$ Ome of miRNAs extracted from Arabidopsis thaliana and mouse lung tissue, as well as piRNA from human seminal plasma.

\section{RESULTS}

\section{Poly(A)-tailed RT-qPCR discriminates 2'-O- methylated miRNA from unmethylated miRNA}

Through comparing the effect of various modifications on the $3^{\prime}$ terminal nucleotide of a small RNA on yeast poly(A) polymerase-catalyzed reaction, Yang et al. (2006) found that among all modifications including 2'dexoxy, 2'Ome, 3'deoxy, and 3'Ome, only 2'Ome significantly inhibited poly(A) polymerase activity. Capitalizing on this finding, we used poly(A)-tailed RT-qPCR to amplify equal amounts of 2'Ome miR-21 and unmethylated miR-21. We compared the result obtained from poly(A)-tailed RT-qPCR with that derived from the standard stem-loop primer RT-qPCR. The schematic diagram of stem-loop primer RT-qPCR and poly(A)-tailed RT-qPCR is shown in Figure 1A. The poly(A)-tailed RT-qPCR results clearly indicated a significant amplification delay on 2'Ome miR-21 compared with unmethylated miR-21, which is opposite to the stem-loop primer RT-qPCR result showing no significant difference between 2'Ome miR-21 and unmethylated miR-21 (Fig. 1B-D).
To exploit the concentration range of these two RTqPCR methods to detect the percentage of $2^{\prime}$ Ome in individual miRNA, we also serially diluted the synthetic miR-21 to concentrations ranging from $10^{-3} \mu \mathrm{M}$ to $10^{-9}$ $\mu \mathrm{M}$, and evaluated the level of amplification with poly(A)tailed RT-qPCR and stem-loop primer RT-qPCR, respectively. Both poly(A)-tailed RT-qPCR and stem-loop primer RT-qPCR showed a linear correlation between $\mathrm{Ct}$ and miRNA concentration (Fig. 1E,F). In this system, the water background, representing a blank control for RT-qPCR, was around $10^{-9} \mu \mathrm{M}$; the minimal concentration of miR21 detected by poly(A)-tailed RT-qPCR was thus about $10 \mathrm{fM}$. The same strategy was applied to the mixture of methylated or unmethylated miR-16 and the result further indicated that poly(A)-tailed RT-qPCR had a higher Ct value when the percentage of miR-16 2'Ome increased (Supplemental Fig. S1).

\section{$\Delta C t$ method for direct quantification of short RNA 2'-O-methylation}

Since poly(A)-tailed RT-qPCR but not stem-loop primer RT-qPCR shows significant amplification delay in methylated miRNAs, we hypothesized that the methylation ratio of a certain miRNA in a certain sample could be detected using these two systems. Therefore, according to the equation:

$$
x=\frac{E_{S}^{C t_{s t d S}-C t_{S}}-E_{T}^{C t_{s t d T}-C t_{T}}}{E_{T}^{C t_{s t d T}-C t_{T}-a}} .
$$

We calculated the methylation ratio by performing RT-qPCR (formula derivation refers to Materials and Methods section) and then plugging $C t$ values into the equation. In this experiment, we mixed synthetic methylated MIR168 (2'Ome MIR168) with unmethylated MIR168 in a different ratio and then detected the mixed samples with both stem-loop primer RT-qPCR and poly(A)-tailed RTGPCR. For MIR168 mixed samples with different methylation ratios, the stem-loop primer $\mathrm{Ct}$ values were almost the same (Fig. 2A), but the tailing $\mathrm{Ct}$ values varied with methylation ratios (Fig. 2B). By plugging the $\mathrm{Ct}$ values into the equation, we can calculate the determined methylation ratio (Fig. 2C). We named this method the $\Delta$ Ct method for methylation ratio detection. Next, we used the same $\Delta \mathrm{Ct}$ method to analyze the percentage of MIR168 methylation in Arabidopsis thaliana. In this experiment, total RNA was extracted from Arabidopsis thaliana. As shown in Figure 2D, we measured the Ct value of MIR168 extracted from Arabidopsis thaliana, synthetic unmethylated MIR168, and 2'Ome MIR168 using stem-loop primer RTqPCR and poly(A)-tailed RT-qPCR, respectively. According to the $\Delta \mathrm{Ct}$ method, we calculated the percentage of MIR168 methylation in Arabidopsis thaliana to be 83\% (Fig. 2E). Serving as either a positive or negative control, 
A

Stemloop primer reverse transcription:

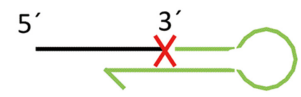

Poly(A)-tailed reverse transcription:

$$
X=2^{\prime} \mathrm{OMe}
$$

$5^{\prime}$<smiles>[Mg][Mg]</smiles>

$\mathrm{X} \operatorname{AAAA}(\mathrm{A})_{n}$ Polyadenylation TTTT T

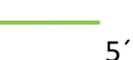

B

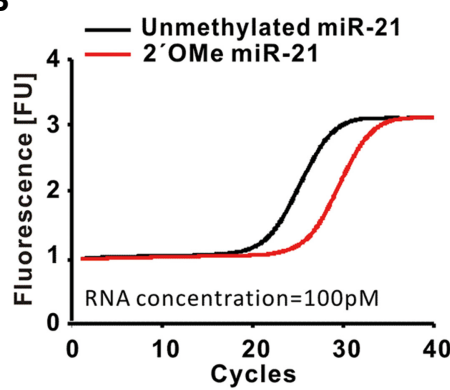

E

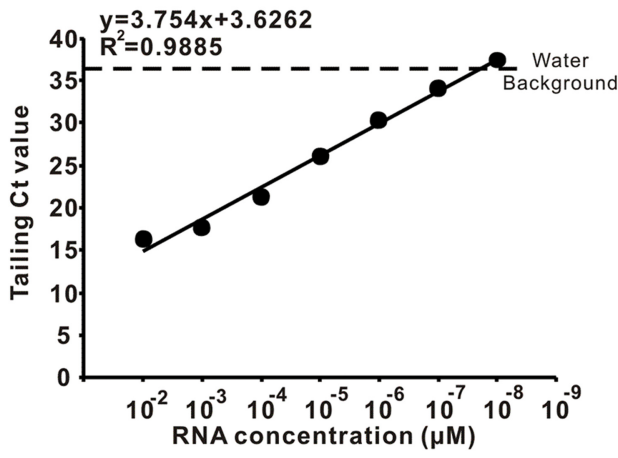

C

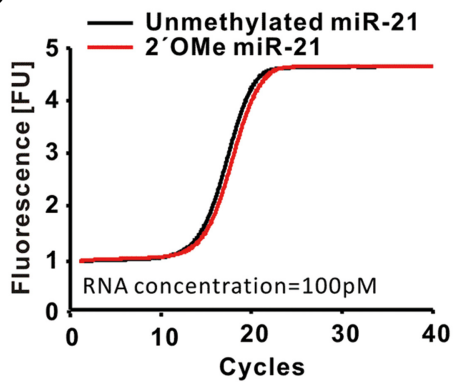

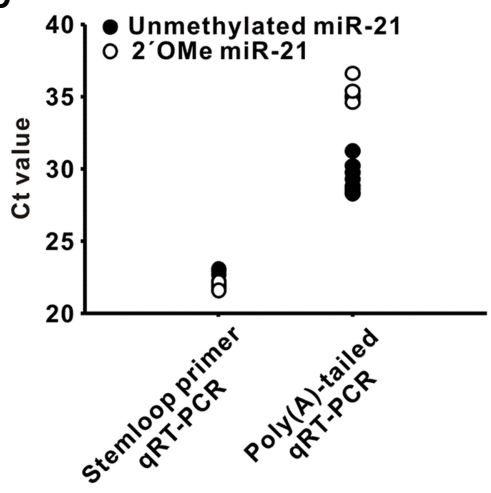

$\mathbf{F}$

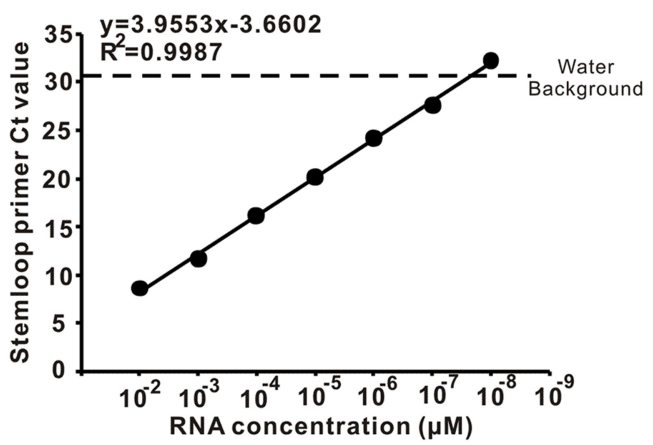

FIGURE 1. Amplification delay on 2'OMe miR-21 by poly(A)-tailed RT-gPCR. (A) Schematic diagram of stem-loop primer reverse transcription and poly(A)-tailed reverse transcription. Black line represents template miRNA. Green line represents the stem-loop primers or universal primers. Red cross represents methylation site. (B) The amplification curve of poly(A)-tailed RT-qPCR for unmethylated miR-21 and 2'Ome miR-21. Note that there is a significant amplification delay by poly(A)-tailed RT-qPCR between unmethylated miR-21 and 2'Ome miR-21. (C) The amplification curve of stem-loop primer RT-qPCR for unmethylated miR-21 and 2'Ome miR-21. Note that there is no significant amplification delay by stemloop primer RT-qPCR between unmethylated miR-21 and 2'Ome miR-21. (D) Ct value of stem-loop primer RT-qPCR and poly(A)-tailed RT-qPCR for unmethylated miR-21 and 2'Ome miR-21. Data were presented as scatter plots $(n=9)$. (E) The standard curve of stem-loop primer RT-qPCR for miR-21. RNA concentration represents the synthetic miR-21 concentration before reverse transcription. $(F)$ The standard curve of poly(A)-tailed RT-qPCR for miR-21. RNA concentration represented the synthetic miR-21 concentration before reverse transcription. In panels $E$ and $F$, the concentration ranges from $10^{-3}$ to $10^{-9} \mu \mathrm{M}$. The upper left shows the regression equation and the goodness-of-fit.

synthetic 2'Ome MIR168 or unmethylated MIR168 showed perfect or negative methylation, respectively.

\section{Nonlinear regression method for direct quantification of miRNA 2'-O-methylation}

Given that plant miRNAs generally are all $3^{\prime}$ terminal methylated, the methylation ratio of $83 \%$ for Arabidopsis thaliana MIR168 detected by our method seemed quite low.
After analyzing the Ct value of various miRNAs for methylation ratio measurement, we observed that the relationship between the Ct value of poly(A)-tailed RT-qPCR and methylation of miRNA fitted more into a second order polynomial curve. As shown in Figure 3A, the relationship between the 2'Ome level of MIR68 and the Ct value of tailed RT-qPCR displayed a perfect second order polynomial curve. The results showed the goodness-of-fit of MIR168 2'Ome versus the Ct value of tailed RT-qPCR by 


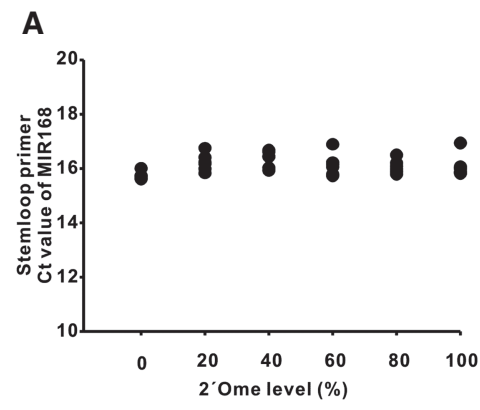

B

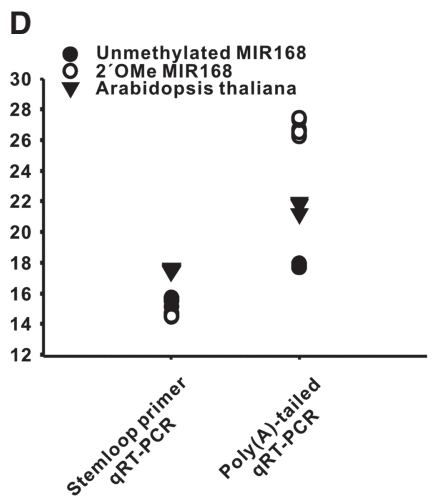

C

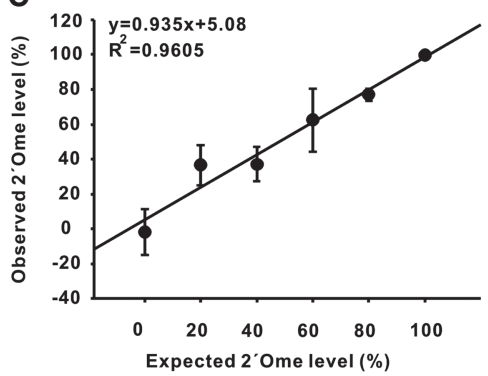

\section{E}

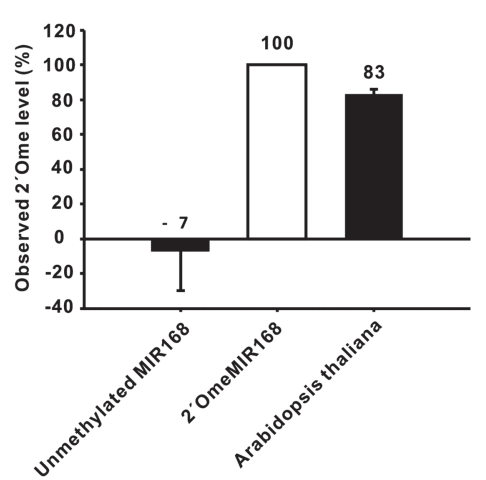

FIGURE 2. Quantification of miRNA methylation ratio by $\triangle$ Ct method. $(A, B)$ Stem-loop primer RT-qPCR (A) and poly(A)-tailed RT-qPCR (B) Ct values of different methylation ratio synthetic MIR168. By substituting $\mathrm{Ct}$ values in the formula, we calculated the methylation ratio for each synthetic sample $(n=6)$. (C) Accuracy of $\Delta$ Ct method in detecting various 2'Ome levels of synthetic MIR168. (D) Stem-loop primer RT-qPCR and poly(A)-tailed RT-qPCR Ct values of MIR168 in Arabidopsis thaliana, synthetic 2'Ome (methylated), and unmethylated MIR168 ( $n=9$ ). (E) Quantification of the methylation ratio of MIR168 in Arabidopsis thaliana by $\Delta$ Ct method. The detected percentage of 2'Ome in MIR168 is indicated on the top of the column. Data are presented as Mean \pm SE $(n=3)$.

nonlinear regression analysis as 0.9953 (Fig. 3B), which was significantly higher than that of linear regression analysis (0.9605, Fig. 2C). We next tested the mixture of synthetic methylated or unmethylated miR-21 and miR-16, and found that the relationship between their 2'Ome levels and $\mathrm{Ct}$ values of tailed RT-qPCR all fit better in a second order polynomial curve (Fig. 3B), suggesting that the second order polynomial curve may be suitable for all short RNAs. To determine the working concentration range of this nonlinear regression analysis, we examined miR-21 at different concentrations. The result showed that the goodness-of-fit of miR-21 2'Ome versus the $\mathrm{Ct}$ value of tailed RT-qPCR was 0.9608 and 0.9834 when the concentration of miR-21 was 100 and 1 pM, respectively (Fig. 3B), suggesting that nonlinear regression analysis has a wide working range of miRNA concentrations. To distinguish from the linear $\Delta \mathrm{Ct}$ method for methylation ratio detection, we named this method as a nonlinear regression method. Next, we further compared the accuracy of these two methods in detecting miRNA 2'Ome levels in a mixture of synthetic methylated and unmethylated miRNAs. As shown in Supplemental Figure S2, the nonlinear regression method (left panels) displayed higher accuracy in measuring the 2'Ome level of MIR168 (100 pM), miR-21
(100 pM), and miR-21 (1 pM) than that of the linear $\Delta \mathrm{Ct}$ method (right panels). A significantly higher goodnessof-fit $\left(R^{2}=0.9935\right.$ for MIR168 and $R^{2}=0.975$ for miR-21) was obtained by the nonlinear regression method compared to that by the $\Delta \mathrm{Ct}$ method $\left(R^{2}=0.8231\right.$ for MIR168 and $R^{2}=0.7756$ for miR-21) when miRNA concentration was 100 pM (Supplemental Fig. S2A,B). Similar results were obtained when miRNA concentration was $1 \mathrm{pM}$ (Supplemental Fig. S2C).

Next, we applied this nonlinear regression method to assess the methylation percentage of individual small RNAs in a real biological sample. In this experiment, synthetic methylated and unmethylated oligonucleotides were mixed according to different ratios as standard samples for establishing the standard curve, respectively. The methylation ratio of MIR168 in Arabidopsis thaliana, miR-16 in mouse lung tissue, and piR-31068 in human seminal plasma was analyzed according to the respective standard curve. In this experiment, the total RNAs from Arabidopsis thaliana, mouse lung tissue, and human seminal plasma were extracted as previously described (Chen et al. 2008; Hong et al. 2016). Based on individual standard curves, we detected the 2'Ome level of MIR168 in Arabidopsis thaliana as $98 \%$ (Fig. 4A). By the same 
A

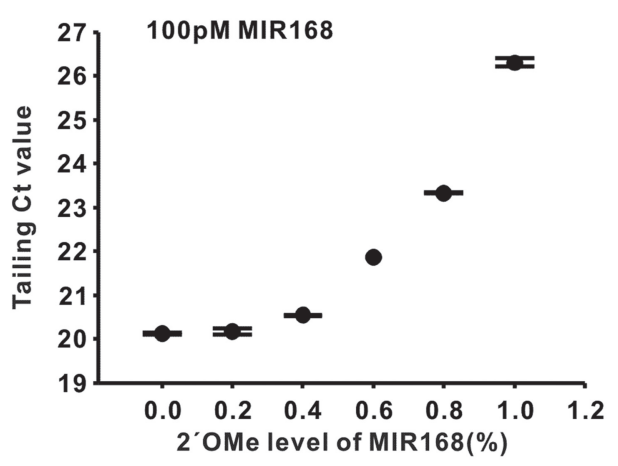

B
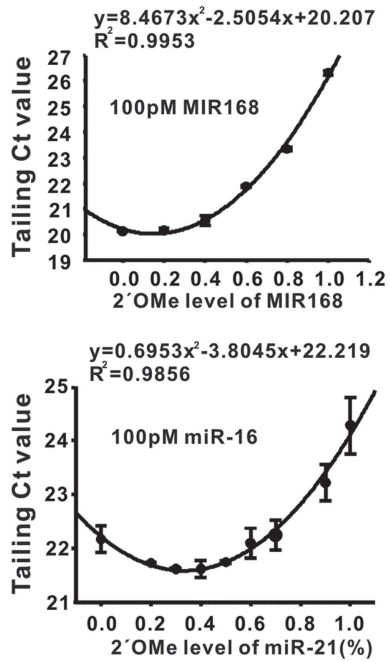
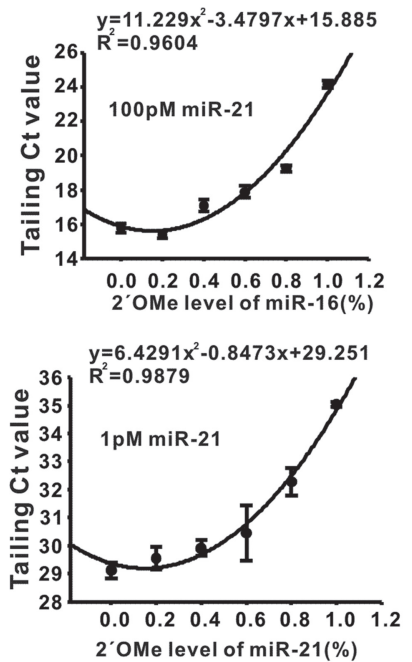

FIGURE 3. Ct value of poly(A)-tailed RT-qPCR and methylation ratio fit of a quadratic curve. (A) Scatter plot of tailing Ct and methylation fraction for synthetic MIR168. The distribution of plots presents a curve. (B) Regression curve of Ct value and methylation ratio for different miRNAs at different concentrations. Upper left is 100 pM MIR168, upper right is 100 pM miR-16, lower left is 100 pM miR-21, and lower right is 1 pM miR-21. Data are presented as Mean $\pm \mathrm{SE}(n=9)$.

method, we obtained the 2'Ome level of miR-21 in mouse lung tissue as $-0.2 \%$ (Fig. 4B) and piR-31068 in human seminal plasma as $99 \%$ (Fig. 4C), respectively. This result suggests that Arabidopsis thaliana MIR168 and human piR-31068 are almost completely 2'-O-methylated at $3^{\prime}$ terminus, whereas mouse miR-21 is not $2^{\prime}-O-$ methylated at all.

It has been shown that the termini of siRNAs and piRNAs in Drosophila are methylated by DmHen1, the Drosophila homolog of HEN1 (Ghildiyal and Zamore 2009). To test whether this direct RT-qPCR method can monitor the alteration of miRNA methylation, we knocked down DmHen1 expression in Drosophila Schneider 2 cells via siRNA. Three DmHen1 siRNA (DmHen1-siRNA1, 2, and 3) and scramble oligonucleotides were obtained and transfected into Drosophila Schneider 2 cells separately or in a combined fashion (DmHen1-siRNA1-3). As shown in Figure $5 \mathrm{~A}$, the expression level of Drosophila DmHen 1 was significantly reduced by three DmHen1 siRNAs but not scramble oligonucleotides. When combining three DmHen1 siRNAs, the Drosophila DmHen1 level in Drosophila Schneider 2 cells was decreased to $30 \%$ of the original level. After extracting RNA from the cells and performing the stem-loop RT-qPCR and tailed RT-qPCR, we calculated the 2'Ome level of Dme-miR-263-5p in various Drosophila Schneider 2 cells by the nonlinear regression method. The result showed that the $2^{\prime}$ Ome level of Dme-miR-263-5p in Drosophila Schneider 2 cells treated with DmHen1siRNA1 or DmHen1-siRNA1-3 was reduced from 103.3 \pm $6.1 \%$ to $73.2 \pm 1.7 \%$ and $49.3 \pm 5.0 \%$, respectively (Fig. 5B). The decrease of the Dme-miR-263-5p 2'Ome level is positively associated with the reduction of DmHen1 in Drosophila Schneider 2 cells.

\section{DISCUSSION}

Accumulating evidence has suggested that plant miRNAs play important roles in various biological processes in not only plants, but also in mammals as exogenous miRNAs derived from food (Carrington and Ambros 2003; Zhang et al. 2012). Given that plant miRNAs, as well as piRNA in animals, are $2^{\prime}-O$-methylated at the $3^{\prime}$ end nucleotide, accurate detection of the $2^{\prime}$-O-methylation of small RNAs is of great importance for further understanding their biological functions. In this study, by capitalizing on the finding that 2'-O-methylation of miRNAs at the $3^{\prime}$ terminal nucleotide can inhibit the activity of poly(A) polymerase, an enzyme essential for RT-qPCR assay, we develop a poly(A)-tailed RT-qPCR method by which the 2'Ome level of small RNAs such as miRNAs and piRNAs can be directly quantified. Using this method, we directly quantified the level of $3^{\prime}$ terminal $2^{\prime}$ Ome of miRNAs extracted from plant and mouse lung tissue, as well as piRNA from human seminal plasma. Furthermore, we successfully monitored the alteration of miRNA 2'-O-methylation in Drosophila Schneider 2 cells after decreasing DmHen-1 expression via DmHen-1-specific siRNA.

The current method for identifying the 2'-O-methylation of small RNA is sodium periodate oxidation combined with northern blot or mass spectrometry (Yu et al. 2005; Ohara et al. 2007). Although these methods are definitive tools to study small RNA methylation, they often require a large amount of RNA and are time-consuming. Therefore, to develop new methods to quantitatively analyze small RNA methylation, several approaches have been pursued. In many cases, these methods are limited to qualitative analysis rather than quantitative analysis of small RNA 
A

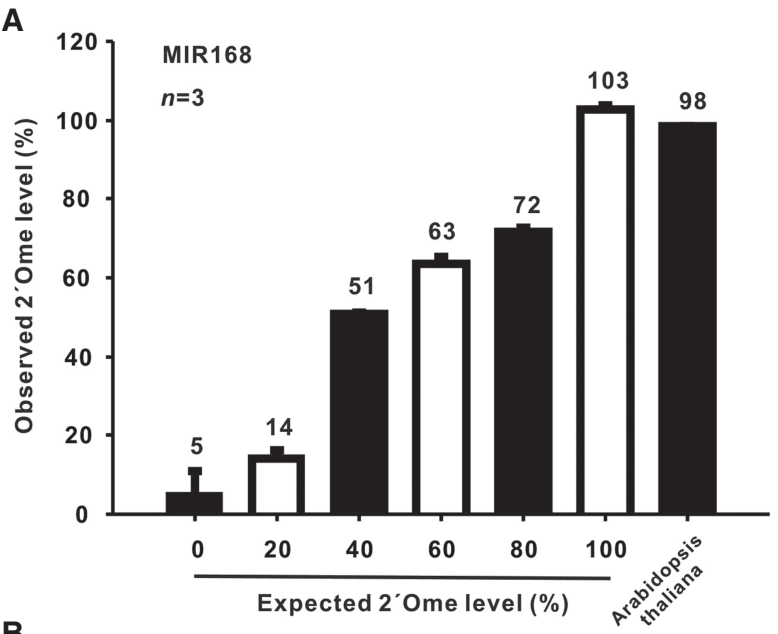

B

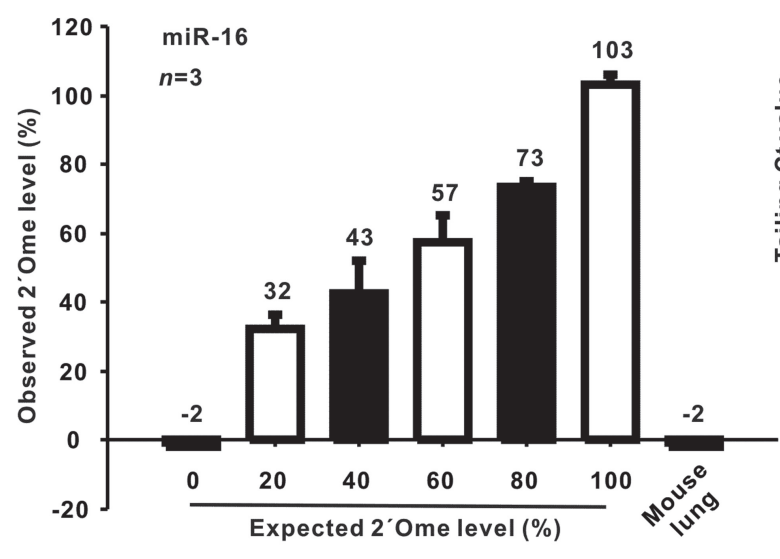

C

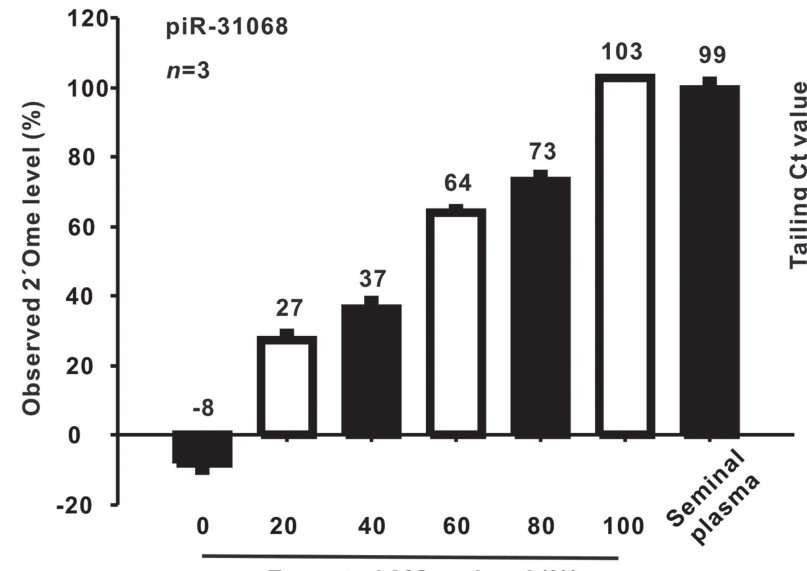

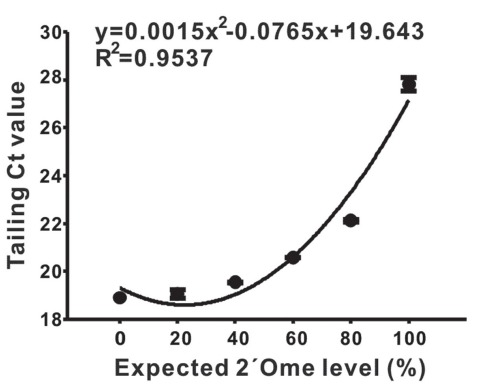

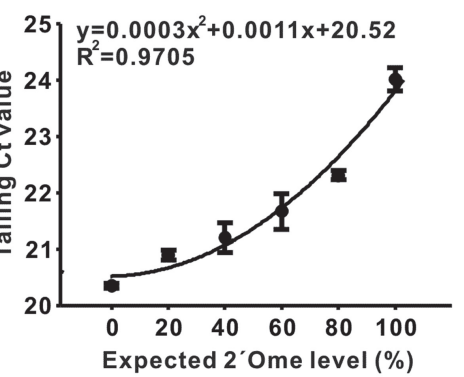

${ }^{36} y=0.0004^{2} x+0.0277 x+27.939$

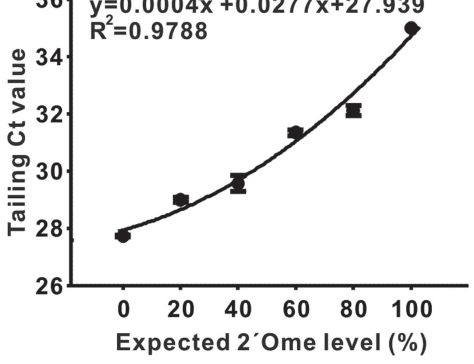

Expected 2'Ome level (\%)

Expected 2'Ome level (\%)

FIGURE 4. The $2^{\prime}$ Ome level of MIR168 in Arabidopsis thaliana, miR-16 in mouse lung tissue, and piR-31068 in human seminal plasma detected by nonlinear regression method. (A) The 2'Ome level of Arabidopsis thaliana MIR168 and synthetic methylated or unmethylated MIR168 detected by nonlinear regression method. (B) The 2'Ome level of mouse lung miR-16 and synthetic $2^{\prime}$ Ome (methylated) or unmethylated miR-16 detected by nonlinear regression method. (C) The 2'Ome level of piR-31068 in human seminal plasma and synthetic methylated or unmethylated piR-31068 detected by nonlinear regression method. In panels A-C, the detected methylation ratios are indicated on the top of the column, while the standard curves of Ct versus methylation for MIR168, miR-16, and piR-31068 are shown in the upper right, respectively. Data are presented as Mean \pm SE ( $n=3$ or 9 as indicated). 
A

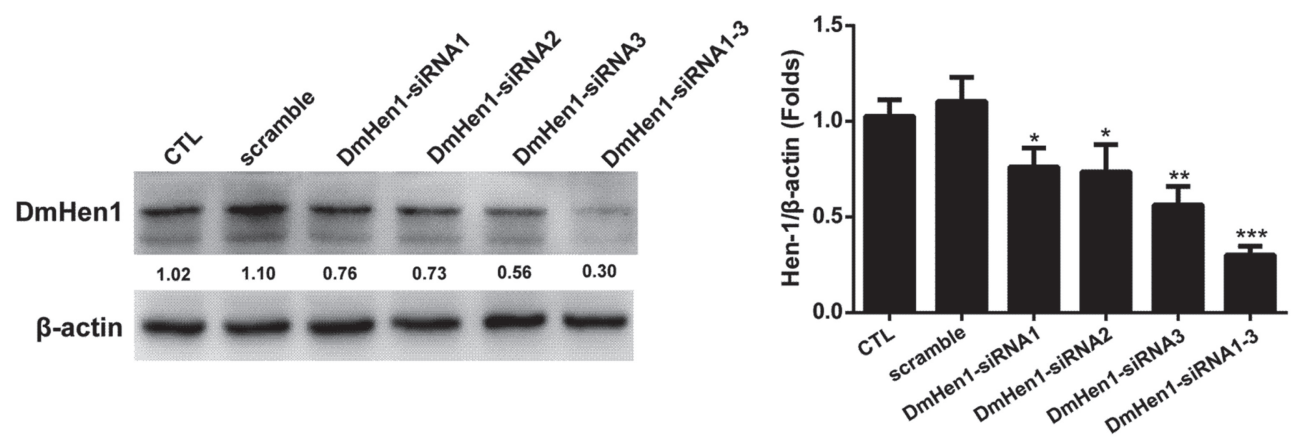

B
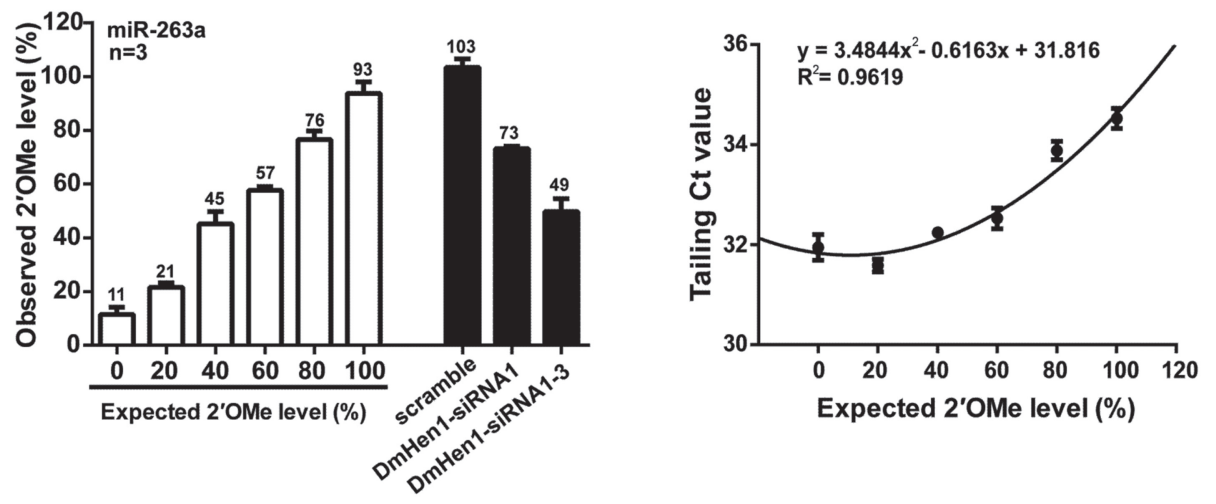

FIGURE 5. The 2'Ome level of Drosophila Dme-miR-263-5p in Drosophila Schneider 2 cells with or without silencing DmHen1. (A) Reduction of DmHen 1 in Drosophila Schneider 2 cells via DmHen 1 siRNAs analyzed by western blotting. (B) The 2'Ome level of Dme-miR-263-5p in Drosophila Schneider 2 cells treated with DmHen1 siRNAs was decreased compared to that in Drosophila Schneider 2 cells treated with scramble oligonucleotides. The detected methylation ratios of Drosophila Dme-miR-263-5p and synthetic methylated or unmethylated Dme-miR-263-5p are indicated on the top of the column, while the standard curves of Ct versus methylation for Dme-miR-263-5p are shown in the upper right. Data are presented as Mean \pm SE $(n=3)$.

methylation. The main problem of quantifying small RNA methylation is the bias against the RNA $3^{\prime}$ terminal 2'-Omethylation by polyadenylate polymerase and T4 RNA ligase, two enzymes that are required for RNA labeling or ligation in commercial miRNA profiling assays. Since polyadenylate polymerase and T4 RNA ligase activity is inhibited by the RNA 3' terminal 2'-O-methylation (Yang et al. 2006; Munafo and Robb 2010), the normal RTqPCR assay with commercial miRNA probes will produce lower detection results of $2^{\prime}$ Ome miRNAs. To minimize this bias related to RNA 3' end 2'-O-methylation, Shen et al. (2012) invented a label-free miRNA profiling method, which makes the high-throughput sequencing more accurate. To overcome the impairment of polymerization reactions for plant miRNAs, which 2'-O-methylation at the $3^{\prime}$ end nucleotide limits their capacity as primers for polymerization reactions, Wang et al. (2017) designed an elegant three-way junction structure-based isothermal exponential amplification reaction (3WJ-EXPAR), which provides a nonbiased technique for detecting plant miRNAs with 3' terminal 2'Ome. Using this 3WJ-EXPAR system, they accurately detected the level of methylated plant miRNAs in laboratory conditions.

In contrast to these approaches, we capitalized on the bias of poly(A) polymerase against $3^{\prime}$ terminal RNA 2'-Omethylation, and developed a poly(A)-tailed RT-qPCR method for directly detecting 2'Ome levels of miRNAs and piRNAs. For individual methylated miRNA or piRNA, poly(A)-tailed RT-qPCR assay results in a higher Ct value than the standard stem-loop miRNA RT-qPCR assay. In fact, more methylation for a small RNA means a larger difference of Ct between two RT-qPCR methods. Therefore, through analyzing the Ct difference we can determine the percentage of $3^{\prime}$ terminal 2'Ome for individual miRNA or piRNA. A similar method has been developed for assaying the methylation level of long RNA fragments such as rRNA (Aschenbrenner and Marx 2016).

Although this direct RT-qPCR assay provides a rapid and accurate method for measuring the level of $3^{\prime}$ terminal $2^{\prime}$ Ome for small RNAs in general, it also has limitations in application. When the $2^{\prime}$ Ome level of individual miRNAs is low, for example, $<20 \%$, the Ct value and methylation 
ratio are not positively correlated. In other words, this method cannot accurately detect samples with a low 2'Ome level. This may be due to the relatively low efficiency of poly (A)-tailed RT-qPCR assays for methylated small RNA. To accurately quantify the low $2^{\prime}$ Ome level of small RNAs, a nonbiased RT-qPCR assay might be required. It is also worth mentioning that this method may not work accurately in quantification of long RNA 3'terminal 2'-O-methylation since our method just discriminates $3^{\prime}$ terminal $2^{\prime}$ Ome and non-2'Ome small RNAs. As internal ribonucleoside 2'Ome modification does significantly influence the activity of poly(A) polymerase by adding poly(A) tails, our method would consider the internal ribonucleoside 2'-Ome modifications as nonmethylated $3^{\prime}$ terminal small RNAs.

\section{MATERIAL AND METHODS}

\section{Synthetic miRNA oligonucleotides and primers}

Synthetic miRNA oligonucleotides include nonmethylated miRNA oligonucleotides, 2'Ome miRNA oligonucleotides (GenePharma) for each miRNA. Primers include stem-loop RT primer, miRNA sense primer, and universal RNA primer (URP) (GenScript) for each miRNA. Oligonucleotides and primers powder were dissolved in DEPC water to generate $20 \mu \mathrm{M}$ and $100 \mathrm{nM} / \mu \mathrm{L}$ stock solution and stored in $-70^{\circ} \mathrm{C}$, respectively. For miRNA methylation detection, the oligonucleotides were diluted into 100 pM concentration before use. For stem-loop primer RT-qPCR calibration, the reaction concentration is $0.1-1.0 \mu \mathrm{M}$. The sequences of all used oligonucleotides and primers are listed in Supplemental Table S1.

\section{RNA extraction}

RNAs were extracted with TRI Reagent (Sigma-Aldrich). A total of 50-100 mg tissue or cell samples were grinded with $1 \mathrm{~mL}$ TRI Reagent in a tissue grinding apparatus. Lung tissues were obtained from 6-wk-old male BalB/c mice. Human semen samples were collected from healthy donors in Jinling Hospital and Nanjing Drum Tower Hospital, Nanjing University with signed consent forms, and the seminal plasma was obtained by centrifuging semen samples at 3000 rpm for 5 min and collecting supernatant. For RNA extraction, $100 \mu \mathrm{L}$ supernatant was mixed with $1 \mathrm{~mL}$ TRI Reagent. After grinding on ice, samples were incubated at room temperature for $5 \mathrm{~min}$, supplemented with $200 \mu \mathrm{L}$ chloroform, vortexed, and centrifuged at $12,000 \mathrm{~g}, 4^{\circ} \mathrm{C}$ for $15 \mathrm{~min}$. The supernatant was transferred to a new $1.5 \mathrm{~mL}$ tube, and then supplemented with $500 \mu \mathrm{L}$ isopropanol and incubated at $-20^{\circ} \mathrm{C}$ for $1 \mathrm{~h}$ followed by centrifugation at $16,000 \mathrm{~g}, 4^{\circ} \mathrm{C}$ for $20 \mathrm{~min}$. After discarding supernatant, the pellet was washed with $1 \mathrm{~mL} 75 \%$ RNase free $\mathrm{EtOH}$, centrifuged again at $16,000 \mathrm{~g}, 4^{\circ} \mathrm{C}$ for $20 \mathrm{~min}$. The pellet was then air-dried and dissolved in 20-50 $\mu \mathrm{L}$ DEPC water.

\section{Cell culture, siRNA transfections, and western blotting}

Drosophila Schneider 2 (S2) cells (ATCC, Schneider's Drosophila Line 2) were cultured in Schneider's Drosophila Medium (Gibco) supplemented with $10 \%$ fetal bovine serum (Gibco) at $25^{\circ} \mathrm{C}$. For transfections, cells were seeded at $8 \times 10^{5}$ cells/well in a 6-well dish, and then cells were transfected with DmHen-1 siRNAs (at a final concentration of $100 \mathrm{nM}$ ) using a Cellfectin II Reagent (Invitrogen). The siRNA sequences are as follows: DmHen1siRNA1: sense: 5'-3' GCUGGAAGGAUCAGAUUAATT, antisense: 5'-3' UUAAUCUGAUCCUUCCAGCTT, DmHen1-siRNA2: sense: 5'-3' GCAAACCCAAU AUCGAUAATT, antisense: 5'-3' UUAUC GAUAUUGGGUUUGCTT, DmHen1-siRNA3: sense: 5'-3' CCUC CCAGGAUGAUGCUUUTT, antisense: 5'-3' AAAGCAUCAUCC UGGGAGGTT. At $48 \mathrm{~h}$ post-transfection, cells were harvested for western blotting. After transfection, cells were harvested and dissolved in RIPA lysis buffer (Beyotime, P0013B) for 30 min on ice. Cell lysates were then centrifuged at $12,000 \mathrm{~g}$ for $20 \mathrm{~min}$ at $4^{\circ} \mathrm{C}$, and the supernatants were harvested. After mixed with loading buffer and denaturation at high temperature, proteins $(30 \mu \mathrm{g} /$ lane) were loaded on $10 \%$ SDS-PAGE gels. After SDS-PAGE separation, the proteins were transferred onto polyvinylidene difluoride membranes. Incubation with primary antibodies was overnight at $4^{\circ} \mathrm{C}$; secondary antibodies were incubated with the membrane for $120 \mathrm{~min}$ at room temperature. The DmHen-1 antibody (Santa Cruz Biotechnology) was used in a 1:500 dilution as a loading control, and $\beta$-actin antibody (Cell Signaling) was used at a 1:1000 dilution. Western blot images were analyzed by Image $\mathrm{J}$ and normalized by $\beta$-actin.

\section{Calibration between nonmethylated and 2'Ome miRNA oligonucleotides}

2'Ome miRNA and unmethylated miRNA oligonucleotides were diluted into the same concentration, and then subjected to stem-loop primer reverse transcription and RT-qPCR detection. There were at least three replicates in each sample. If the difference value for $\mathrm{Ct}$ between two samples is smaller than 0.2 , we will consider the calibration is completed. If not, we continue diluting the higher concentration sample according to the $\mathrm{Ct}$ value until the Ct difference is smaller than 0.2.

\section{Formula derivation}

According to the equation:

$$
\text { Methylation fraction }=1-E^{-\left(\Delta C_{T 1}-\Delta C_{T 2}\right)},
$$

with $E=P C R$ efficiency (in an ideal situation, $E=2$ ). In this equation, $\Delta \mathrm{C}_{\mathrm{T} 1}$ and $\Delta \mathrm{C}_{\mathrm{T} 2}$ represent stem-loop $\mathrm{RT}$-qPCR and poly(A)tailed RT-qPCR systems, respectively. To obtain more accurate results, we refined an equation as follows:

$$
\frac{E_{S}^{C t_{s t d S}-C t_{S}}}{E_{T}^{C t_{s t d T}-C t_{T}}}=(1-x)+x \cdot E_{T}^{-a},
$$

in which

$E_{S}=$ stem-loop PCR efficiency;

$\mathrm{E}_{\mathrm{T}}=$ tailing $\mathrm{PCR}$ efficiency;

$\mathrm{x}=$ methylation fraction;

$\mathrm{a}=\Delta \mathrm{Ct}$ between synthetic methylated miRNAs and equal amounts of unmethylated miRNAs with poly(A)-tailed RTqPCR; 
$\mathrm{Ct}_{\text {stds }}=\mathrm{Ct}$ value of synthetic oligonucleotide with stem-loop primer PCR (the synthetic oligonuleotides concentration is in the same level with the sample to be detected);

$\mathrm{Ct}_{\text {stdT }}=\mathrm{Ct}$ value of synthetic oligo nucleotide with poly(A)-tail PCR;

$\mathrm{Ct}_{\mathrm{S}}=\mathrm{Ct}$ value of stem-loop primer RT-qPCR for the sample to be tested;

$\mathrm{Ct}_{\mathrm{T}}=\mathrm{Ct}$ value of poly(A)-tailed RT-qPCR for the sample to be tested.

Therefore, $x$ can be derived through the solution of this equation:

$$
x=\frac{E_{S}^{C t_{\text {stdS }}-C t_{S}}-E_{T}^{C t_{\text {std }}-C t_{T}}}{E_{T}^{C t_{\text {stdT }}-C t_{T}-a}} .
$$

\section{RT-qPCR for stem-loop primer method and tailing method}

There were two systems of RT-qPCR in our research: stem-loop primer system and tailing system. For the stem-loop primer system, which was used for calibration, the reverse transcription system included $0.25 \mathrm{U} / \mu \mathrm{L}$ AMV Reverse transcriptase XL (Takara), $0.25 \mathrm{mM}$ dNTP mixture (Takara), and $0.25 \mu \mathrm{M}$ stem-loop RT primer according to the vendor's protocol. Samples were kept on ice before running programs. The reverse transcription program was as follows: $16^{\circ} \mathrm{C}$ for $15 \mathrm{~min}, 42^{\circ} \mathrm{C}$ for $30 \mathrm{~min}$, and $85^{\circ} \mathrm{C}$ for $5 \mathrm{~min}$. The real-time quantitative PCR system was $0.07 \mathrm{U} / \mu \mathrm{L} r \mathrm{rTaq}$ (Takara), 0.2 mM dNTP (Takara), $1 \times$ EvaGreen (Invitrogen), 0.125 $\mu \mathrm{M}$ sense primer, $0.125 \mu \mathrm{M}$ URP, $1.5 \mathrm{mM} \mathrm{MgCl}_{2}, 1 \times \mathrm{PCR}$ buffer $\left(\mathrm{Mg}^{2+}\right.$ free). The program was as follows: $95^{\circ} \mathrm{C}$ for $5 \mathrm{~min}$, followed by 40 cycles of $95^{\circ} \mathrm{C}$ for $15 \mathrm{sec}$ and $60^{\circ} \mathrm{C}$ for $1 \mathrm{~min}$. Each cDNA sample was tested in triplicate. For the tailing system, which was used for detecting methylation proportion in synthetic oligonucleotides as well as sample RNAs, All-in-One miRNA FirstStrand cDNA Synthesis Kit (GeneCopoeia), containing poly(A) polymerase and RTase Mix, was used according to the manufacturer's instructions. After obtaining cDNAs, rTaq (Takara), dNTP (Takara), EvaGreen (Invitrogen), sense primer, and Universal Adaptor PCR Primer (GeneCopoeia) were used for RT-qPCR detection. The program was as follows: $95^{\circ} \mathrm{C}$ for $5 \mathrm{~min}$, followed by 40 cycles of $95^{\circ} \mathrm{C}$ for $15 \mathrm{sec}$ and $60^{\circ} \mathrm{C}$ for $1 \mathrm{~min}$. Each cDNA sample was tested in triplicate.

\section{Nonlinear regression analysis and calculation of methylated proportion}

After obtaining tailing $\mathrm{Ct}$ values for different methylation ratio synthetic oligonucleotides, the Ct values of miRNAs and their corresponding methylation proportion were fitted into a second order polynomial curve (Excel, Microsoft), such as $y=a x^{2}+b x+c$, in which $y$ and $x$ represent $C t$ value and methylation proportion, respectively. $\mathrm{X}$ was then calculated according to the formula.

\section{SUPPLEMENTAL MATERIAL}

Supplemental material is available for this article.

\section{ACKNOWLEDGMENTS}

The authors thank Drs. Jill Leslie Littrell and Koby Shane Kidder (Georgia State University, Atlanta, GA) for critical reading and constructive discussion of the manuscript. This work was supported by grants from the National Key R\&D Plan (2018FA0507100), the National Natural Science Foundation of China (91640103, 31670917, 31770981), Natural Science Foundation of Jiangsu Province (BK20170076), Six Talents Peak Project of Jiangsu Province, and Fundamental Research Funds for the Central Universities (020814380095, 020814380082).

Received November 30, 2017; accepted July 25, 2018.

\section{REFERENCES}

Ameres SL, Horwich MD, Hung JH, Xu J, Ghildiyal M, Weng Z, Zamore PD. 2010. Target RNA-directed trimming and tailing of small silencing RNAs. Science 328: 1534-1539.

Aschenbrenner J, Marx A. 2016. Direct and site-specific quantification of RNA 2'-O-methylation by PCR with an engineered DNA polymerase. Nucleic Acids Res 44: 3495-3502.

Backes S, Shapiro JS, Sabin LR, Pham AM, Reyes I, Moss B, Cherry S, tenOever BR. 2012. Degradation of host microRNAs by poxvirus poly(A) polymerase reveals terminal RNA methylation as a protective antiviral mechanism. Cell Host Microbe 12: $200-210$.

Carrington JC, Ambros V. 2003. Role of microRNAs in plant and animal development. Science 301: 336-338.

Chen X, Ba Y, Ma L, Cai X, Yin Y, Wang K, Guo J, Zhang Y, Chen J, Guo $X$, et al. 2008. Characterization of microRNAs in serum: a novel class of biomarkers for diagnosis of cancer and other diseases. Cell Res 18: 997-1006.

Cohn WE. 1960. Pseudouridine, a carbon-carbon linked ribonucleoside in ribonucleic acids: isolation, structure, and chemical characteristics. J Biol Chem 235: 1488-1498.

Ghildiyal M, Zamore PD. 2009. Small silencing RNAs: an expanding universe. Nat Rev Genet 10: 94-108.

Hong $Y$, Wang C, Fu Z, Liang H, Zhang S, Lu M, Sun W, Ye C, Zhang CY, Zen K, et al. 2016. Systematic characterization of seminal plasma piRNAs as molecular biomarkers for male infertility. Sci Rep 6: 24229.

Horwich MD, Li C, Matranga C, Vagin V, Farley G, Wang P, Zamore PD. 2007. The Drosophila RNA methyltransferase, DmHen1, modifies germline piRNAs and single-stranded siRNAs in RISC. Curr Biol 17: 1265-1272.

Ji L, Chen X. 2012. Regulation of small RNA stability: methylation and beyond. Cell Res 22: 624-636.

Kirino Y, Mourelatos Z. 2007a. 2'-O-methyl modification in mouse piRNAs and its methylase. Nucleic Acids Symp Ser (Oxf) 51: 417-418.

Kirino Y, Mourelatos Z. 2007b. Mouse Piwi-interacting RNAs are 2'O-methylated at their $3^{\prime}$ termini. Nat Struct Mol Biol 14: 347-348.

Li J, Yang Z, Yu B, Liu J, Chen X. 2005. Methylation protects miRNAs and siRNAs from a $3^{\prime}$-end uridylation activity in Arabidopsis. Curr Biol 15: 1501-1507.

Limbach PA, Crain PF, McCloskey JA. 1994. Summary: the modified nucleosides of RNA. Nucleic Acids Res 22: 2183-2196.

Machnicka MA, Milanowska K, Osman Oglou O, Purta E, Kurkowska M, Olchowik A, Januszewski W, Kalinowski S, DuninHorkawicz S, Rother KM, et al. 2013. MODOMICS: a database of RNA modification pathways-2013 update. Nucleic Acids Res 41: D262-D267. 
Motorin Y, Helm M. 2011. RNA nucleotide methylation. Wiley Interdiscip Rev RNA 2: 611-631.

Munafo DB, Robb GB. 2010. Optimization of enzymatic reaction conditions for generating representative pools of cDNA from small RNA. RNA 16: 2537-2552.

Ohara T, Sakaguchi Y, Suzuki T, Ueda H, Miyauchi K, Suzuki T. 2007. The $3^{\prime}$ termini of mouse Piwi-interacting RNAs are 2'-O-methylated. Nat Struct Mol Biol 14: 349-350.

Saito K, Sakaguchi Y, Suzuki T, Suzuki T, Siomi H, Siomi MC. 2007. Pimet, the Drosophila homolog of HEN1, mediates 2'-O-methylation of Piwi- interacting RNAs at their $3^{\prime}$ ends. Genes Dev 21: 1603-1608.

Shen Y, Zheng KX, Duan D, Jiang L, Li J. 2012. Label-free microRNA profiling not biased by $3^{\prime}$ end 2'-O-methylation. Anal Chem 84: 6361-6365.
Wang X, Wang H, Liu C, Wang H, Li Z. 2017. A three-way junction structure-based isothermal exponential amplification strategy for sensitive detection of 3'-terminal 2'-O-methylated plant microRNA. Chem Commun 53: 1124-1127.

Yang Z, Ebright YW, Yu B, Chen X. 2006. HEN1 recognizes 21-24 nt small RNA duplexes and deposits a methyl group onto the $2^{\prime} \mathrm{OH}$ of the $3^{\prime}$ terminal nucleotide. Nucleic Acids Res 34: 667-675.

Yu B, Yang Z, Li J, Minakhina S, Yang M, Padgett RW, Steward R, Chen X. 2005. Methylation as a crucial step in plant microRNA biogenesis. Science 307: 932-935.

Zhang L, Hou D, Chen X, Li D, Zhu L, Zhang Y, Li J, Bian Z, Liang X, Cai $X$, et al. 2012. Exogenous plant MIR168a specifically targets mammalian LDLRAP1: evidence of cross-kingdom regulation by microRNA. Cell Res 22: 107-126. 

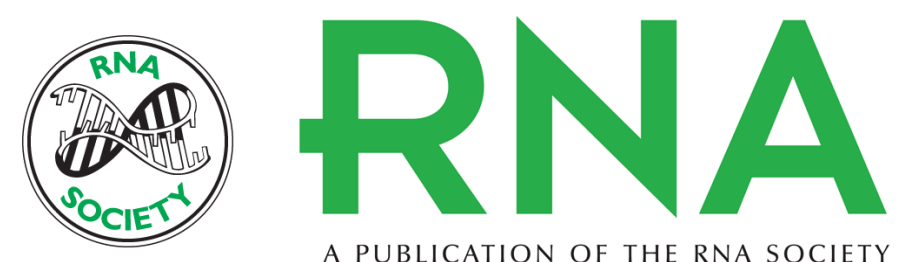

A PUBLICATION OF THE RNA SOCIETY

\section{Direct quantification of 3' terminal 2'-O-methylation of small RNAs by RT-qPCR}

Nan Wang, Shuang Qu, Wu Sun, et al.

RNA 2018 24: 1520-1529 originally published online August 3, 2018

Access the most recent version at doi:10.1261/rna.065144.117

\section{Supplemental Material}

References

Creative Commons License

Email Alerting
Service
http://rnajournal.cshlp.org/content/suppl/2018/08/02/rna.065144.117.DC1

This article cites 24 articles, 6 of which can be accessed free at: http://rnajournal.cshlp.org/content/24/11/1520.full.html\#ref-list-1

This article is distributed exclusively by the RNA Society for the first 12 months after the full-issue publication date (see http://rnajournal.cshlp.org/site/misc/terms.xhtml). After 12 months, it is available under a Creative Commons License (Attribution-NonCommercial 4.0 International), as described at http://creativecommons.org/licenses/by-nc/4.0/.

Receive free email alerts when new articles cite this article - sign up in the box at the top right corner of the article or click here.

To subscribe to $R N A$ go to:

http://rnajournal.cshlp.org/subscriptions 